\title{
Astronomy Research via the Internet
}

\author{
Kavan U. Ratnatunga \\ Dept. of Physics, Carnegie Mellon University, Pittsburgh, PA 15213, \\ U.S.A.e-mail: kavan@astro.phys.cmu.edu
}

\begin{abstract}
.
Small developing countries may not have a dark site with good seeing for an astronomical observatory or be able to afford the financial commitment to set up and support such a facility. Much of astronomical research today is however done with remote observations, such as from telescopes in space, or obtained by service observing at large facilities on the ground. Cutting-edge astronomical research can now be done with low-cost computers, with a good Internet connection to get on-line access to astronomical observations, journals and most recent preprints. E-mail allows fast easy collaboration between research scientists around the world. An international program with some short-term collaborative visits, could mine data and publish results from available astronomical observations for a fraction of the investment and cost of running even a small local observatory. Students who have been trained in the use of computers and software by such a program would also be more employable in the current job market. The Internet can reach you wherever you like to be and give you direct access to whatever you need for astronomical research.
\end{abstract}

\section{Introduction}

The exponential growth of astronomical research in the twentieth century, has left the developing nations far behind in contributions to the field. Pure science research in all non-theoretical fields has required very expensive technology which cannot be justified in the absence of immediate practical benefit to the developing nation.

There is a tendency to assume that astronomers in developing countries need to limit themselves to do astronomy research with less expensive small telescopes. Many talks at this Special Session discuss many of the basic but useful astronomical observations being done at small observatories around the world.

Let me first state clearly to avoid being misunderstood that I am not in anyway doubting the role that small telescopes can play in astronomical research or in inspiring students to study astronomy. Continuous monitoring of planets, comets, variable stars and other transient astronomical events benefit significantly from a global network of telescopes. Many developing nations could fill important gaps in such coverage. For example my home nation of Sri Lanka 
could fill the southernmost latitude between the longitudes of South Africa and Western Australia, north of Antarctica.

The astronomical research environment has however changed over the last five years in many significant ways. The price of computers needed for data analysis and cost of on-line data storage has dropped tenfold. The amount of astronomical data available in on-line archives from both ground-based and spaced-based observations is growing exponentially. Submission of papers to most leading journals is done on-line. Practically all leading astronomy journals back to their first volumes are now archived at Astrophysics Data System (ADS http://adsabs.harvard.edu/). The latest preprints are also archived at astro-ph (http://xxx.lanl.gov/) and updated daily.

The point I wish to make is that an on-site local telescope is now not need to start active research and education in astronomy. For example, Hubble SpaceTelescope observations (HST http://archive.stsci.edu/) are released after one year and images taken in parallel mode become freely available on the Internet a day after the observations are made. Everyone on the Internet has equal opportunity to make and publish discoveries from them.

Cutting-edge astronomical research can therefore be done from any place in the world. All that is needed are low-cost computers with a good Internet connection to get on-line access to astronomical, observations, journals and most recent preprints. Since the investment in Internet connectivity is now justified, not only by research, but by e-commerce it is simpler for developing countries to obtain financial aid to establish such network connectivity, which then helps scientific research and the economy in many ways.

I will discuss below, in order of increasing importance, what I consider to be the main advantages of doing astronomy research over the Internet, pointing out the opportunities and limitations. Finally I will discuss as an example the situation in my home nation Sri Lanka, where enthusiasm and spurts of investment in conventional astronomy, have as yet, unfortunately, not yielded any significant research progress.

\subsection{Independent of site}

Astronomical observational sites with good seeing are rare. Urban growth and accompanying light pollution make it increasingly difficult to find a reasonable dark site. A remote site, even if one exists, leads to practical logistical problems of transport and accommodation. Access roads and guest-observer support are substantial additions to the cost. This has led to many small telescopes in developing countries being installed in bright urban areas and remaining practically useless for astronomical research.

However, the Internet can reach you wherever you like to be and give you direct access to whatever you need for astronomical research. For example, Sir Arthur C. Clarke, who predicted global communications based on geo-stationary satellites, has chosen to make Sri Lanka his home. The Internet connects him to the rest of the global village. How many academics, particularly expatriates, in all fields could be encouraged to return or to spend a productive sabbatical in their developing home country if network access needed for efficient communications and research were to be set up! In the presence of truly global communications there is no need to continue to exile oneself in a foreign land. 


\subsection{Not dependent on weather}

Bad weather seems always to follow you even at good sites. The beauty of the solar corona watched with the naked-eye during a total eclipse of the Sun is not describable by words or recorded on film. It has to be seen to be appreciated. I was at the driest spot in the world, the Atacama desert in Bolivia, to observe my first total eclipse of the Sun in November 1994. It rained the night before!

I agree there is clearly a vast difference in experiencing personal observations compared with seeing images in a book or getting digital data over the Internet. Even relatively bright Messier objects through a small telescope may-be a disappointment in comparison to seeing all those beautiful long exposure image processed photographs of them on the Internet (http://www.seds.org/messier/).

Cloud cover often dampens one's enthusiasm to continue regular observations, particularly of transient events. The consequent lack of observations could lead to a complete loss of productivity when one tries to start an active research program.

However, serious quantitative astronomy research is not now done with observations made at an eyepiece. Is there any difference if the observations come from the instruments on a local telescope or come to your computer over the Internet from a telescope far away?

An astronomy research program set-up which includes the analysis of remote observations is obviously not dependent on the local weather and need not be interrupted even if one is temporarily disconnected from the Internet. No program needs to be data starved. There are far more astronomical data with free on-line access than it is humanly possible for the original observers to look at.

\subsection{No need for expensive instrumentation}

Telescopes need to be equipped with more than a set of eyepieces for any observations to be used for quantitative astronomical research. Telescopes need special instrumentation such as image detectors and spectrographs. Some spectral regions (e.g., infrared) require expensive cryogenics. Investing in and maintaining an expensive observatory which is useful only for astronomers can be unaffordable for a developing country.

All you need to get started on astronomy research over the Internet is a low-cost personal computer and a link to the Internet. Most of the major software analysis packages are freely available. Unlike costly instrumentation, the development of software requires only skill, a compiler, and a time commitment.

Astronomy resources on the Internet change over time and any detailed list would at most be a snapshot. In the appendix I list some of the major sites probably biased by my personal preference.

AstroWeb (http://cdsweb.u-strasbg.fr/astroweb.html) is an astronomical Internet Resources database which has been maintained since 1994 by a small world-wide consortium of institutions and contains pointers to potentially relevant resources available via Internet. There are a number of mirrors of this site around the globe. It is a good starting place to find anything related with astronomy. It provides searchable links to find telescope-time applications, data centers with astronomy archives, on-line astronomy journals, observation-analysis software, astronomy-conference information etc. It is not based on indexing au- 
tomated downloads of web-sites. It is a moderated data-base which ensures that you don't need to search through a lot of unimportant URL's which happened to match search keywords to find the most useful sites which match your query. It depends on authors to submit to the site and, therefore, if you have set up an Internet web site associated with astronomy, then you should add it on-line to this database. Another advantage of this site is that an automated Tcl script checks the validity of the links every day to warn users of any that are broken.

\subsection{Remote observing}

Service observations are now common since they are a more efficient way to share time on a large-telescope facility. With space-based telescopes there is no choice. As instrumentation becomes more complex a local expert is in any case needed to set it up. Most often the required observations can easily be predefined precisely with exposure times or signal required. Telescope-floor decisions are typically made to use available time slots, often degraded by weather, to optimize which observations are taken. This is not the case for service observations which are aranged in a queue and made whenever the weather, seeing and photometric conditions required for each program are satisfied. Service observing obivates the need for costly and time-consuming travel to an observatory, from which one often returns with no data because of bad weather. If active interaction is important you can even observe remotely over the Internet. This could even be during the day, if you are using a telescope half-way across the world.

For example, scientists of any nationality or affiliation may submit to NASA Hubble-Space-Telescope (HST http://www.stsci.edu/) proposals. Restrictions are only for funding requests. All you need is to submit excellent observational proposals over the Internet to win the telescope time required. For other facilities all one needs is at most a collaboration with an observer with access to that telescope. The observations are made as requested and the data are sent by tape or can be obtained over the Internet.

\subsection{Rapid decrease in cost of computers}

Twenty years ago astronomical data-analysis was done on large VAX/VMS computers which cost over U.S. $\$ 100,000$. Only large departments or universities as a whole could afford them. Users shared time on this central computer via terminals. Most developing nations could not afford them without significant grants. Ten years ago the same power was available on SUN/UNIX workstations which cost over U.S. $\$ 10,000$ but started to become personal desk-top units. Today personal computers are more than 10 times powerful and cost under U.S. $\$ 1000$. Computer hard-disk space is also important for astronomy data-analysis. Ten years ago it cost over U.S. $\$ 2000$ per GB. Today disk storage costs under U.S. $\$ 10$ per GB.

Increasingly, in many academic environments, the free shareware UNIX operating system known as LINUX has enabled home-market personal computers to replace the more costly workstations without sacrificing software portability, numerical computing power or operational stability.

Computers depreciate in value very fast. So investment in the needed computer and data storage should be made only as the research program grows. A computer laboratory has a half-life of under 2-years. A computer laboratory set 
up five years ago would probably be of no residual value. In contrast a telescope will, in principle, have an operational life-time of probably over 50 years, if it is set up and used in a good site, and still retain its value.

However, computer costs have dropped so much that they are now small compared to operational costs such as salary, and travel. The cost of computers and disk storage are clearly not limiting factors in setting up a computer laboratory for astronomical research.

Good Internet connectivity is also becoming affordable although it is still a non-negligible cost for a developing nation. I read on the web that connectivity with T1 bandwidth (1.5Mbits/sec) costs about $\$ 1000$ per month in the U.S. and is sufficient for only about 75 users. Although telecoms may charge ten times this figure, in reality it is no more expensive to a government of a developing nation. A T3 (28Mbits/sec) line would be needed to download files of order $100 \mathrm{MB}$ or more in reasonable time.

\subsection{Common research facilities}

The set-up of computer facilities and Internet connectivity are common to most fields of scientific research. It is now also used by e-commerce and the public at large. This is a very important consideration when it is proposed to start any fundamental scienctific research like astronomy in developing countries, where everything needs to be related to practical needs of the country. Astronomy is clearly useful for the development of the intellectual environment but we must recognize that it is a luxury for which it is probably difficult to justify independent funding.

Computers serve both the analysis of observations and theoretical studies. E-mail allows easy collaboration with research scientists around the world. Voice and even video-phone calls over the Internet bring added possibilities for fast and inexpensive communication. However for active research, one needs to be able to get fast response during working hours for remote interactive log-in and to have the ability to transfer of the order of $100 \mathrm{MB}$ or more on demand, without unreasonable time delays. Developing nations need to negotiate with funding agencies to obtain seed money to set up collaborative efforts for analysis of observations and data mining.

\subsection{Job security for students}

A Ph.D. in astronomy does not ensure a research or teaching position. In reality many good post-doctoral fellows, even in the West, need to switch to nonastronomy jobs. Other than as an indicator of a level of intellectual maturity, expertise in pure astronomy has no practical applications in commercial industry. Students familiar with computer data-analysis and the Internet, however, find it much simpler to find gainful employment. The growth of the Internet is so rapid that for a long time there will ample Internet-related jobs. The type of expertise one gains while doing research is important. A person with a bachelor's degree and good computer skills is probably more employable than one with a Ph.D. in astronomy but without advanced computer capabilities. 


\subsection{Extensive on-line archives of journals.}

The Internet has more astronomy journals on-line than most major university libraries including Carnegie Mellon have on their shelves. Astronomy journals back to their first issues are now archived digitally at Astrophysics Data System (ADS) at http://adsabs.harvard.edu with mirror sites around the globe.

No longer do you need to suffer months of delay waiting for journals to arrive, posted by sea-mail because of excessive air-mail costs. Everyone on the Internet can see publications at the same time. Electronic submission of papers to practically all journals reduces delays and postage costs in the referee and publication process. It has also minimized the effort needed for proof corrections since the submitted manuscripts in $\mathrm{IAT}_{\mathrm{E}} \mathrm{X}$ or other standard word-processor format are directly translated to the format needed for publication.

The latest preprints are also archived at astro-ph (http://xxx.lanl.gov) and updated daily. New abstracts are sent out by e-mail. Since most astronomers now read the latest papers from this free service it has almost replaced the costly distribution of preprints. Submission to the astro-ph server is now considered an essential part of publication, to ensure that a paper will come to the attention of the astronomy research community.

However, it is important to negotiate with the on-line journal publishers to allow access to recent on-line editions for developing nations without institutions being required to subscribe to the expensive printed copy. Institutions should also have access to commercial products such as the ISI's Web of Science, a journal-article data-base that covers more than 5300 major journals in the natural sciences, mathematics, engineering, technology, and medicine.

\subsection{Leading instruments and telescopes}

Observations taken with the leading instruments like the Hubble Space Telescope and large ground-based national facilities can be down-loaded on the Internet. A recent National Academy of Science report, Astronomy and Astrophysics in the New Millennium, discusses a virtual observatory as a very important initiative in astronomy over the next decade; a digital sky in all wavelengths based on the massive data sets being created with tools to explore the data base.

All space-based missions and most large ground-based observatories maintain a complete data archive of observations. Almost all HST observations are put in the public domain after one year. However you are not limited to astronomical observations which have been milked for the best science. Pure-Parallel observations made with the HST are put on-line the day after observation, free for an astronomer from anywhere in cyberspace to analyze.

\subsection{Archived data available freely}

Large ground-based and space-based surveys and individual observations are generating more data than most observers can use, except for the very specific application for which the observations were made. There is a lot more invaluable astronomical information in most of these observations, for different applications, if one has the time to look more carefully. 
There is a lot of archived data on-line with free Internet access, much of which has not been analyzed in detail, nor even been looked at in detail, because the original observers had insufficient funding.

For example, the first gravitational lens to be discovered by the Hubble Space telescope was found by us as a part of the Medium Deep Survey (MDS) on archival data (Ratnatunga et al. 1995), after the observations were released to the public one year after they had been made. The only advantage that the MDS group had was the software we had developed to handle automatically some aspects of the data reduction (Ratnatunga et al. 1996). Software like this could be easily obtained in collaboration or even developed without large investment. However much of our discovery was due to the fact that, in the pipeline of automated analysis, we included in addition a careful visual inspection of the observations. The trained human eye is still far better than any software in picking out the interesting and unusual objects, and far less visual inspection is done now than should be - a lapse that any developing country properly connected to the Internet can exploit.

There are many opportunities to develop programs for data mining. It is much less expensive than data acquisition and the credit of discovery is with the person who publishes the results not with the person who made the observation. There are probably lots of hidden serendipitous discoveries to be made in spacebased observational data obtained at astronomical cost.

\section{An example: Sri Lanka}

Astronomical research in Sri Lanka is probably typical of that in many developing countries. I use Sri Lanka as an example to illustrate how, like many other developing nations, it could develop more by the Internet than with a small telescope. I have observed first hand its attempts to develop in astronomy for over 30 years.

The absence of astronomical research in Lanka is not as Gehrels (1988) suggested in his review of Sri Lanka's Telescope (I quote) "Could it be that inquiry into our origins has little appeal to Buddhists ?". Questioning authority and the status quo is a cornerstone of Buddhism as the Kalama Sutta (http://www.accesstoinsight.org/lib/bps/wheels/wheel008.html) expresses very eloquently. This was the Eastern experience of the Renaissance and the cultural attitudes, such as a willingness to confront authority and reject religion which Snowden (2001) feels is now lacking in Sri Lanka. In any case, only a minute fraction of the population in the West or the East are associated with research in fundamental science such as astronomy, the mind-set of the current population at large is irrelevant to the establishment of astronomy research programs. For example, $47 \%$ of Americans- and a quarter of college graduates -believe humans did not evolve, but were created by God a few thousand years ago, and yet the U.S.A. is the world's leading scientific nation. (MacKenzie 2000).

Eastern civilization in particular has had an interest in the cosmos from ancient times. They accepted the vastness of space and time while religious belief in the West adopted a geocentric universe created 6000 years ago. Hindus for example believed in a cyclic universe with a period of 7.3 billion years. I quote from the 1822 book Hindoostan edited by Frederic Shoberl "A learned Bramin 
laughed, on being told that we Europeans reckon only about six thousand years since the creation of the world, and pointing to an old man with a long beard, asked if it was possible to believe that he was born but the preceding day."

Astronomical research in Sri Lanka dates back to the early 1900s when Major P. B. Molesworth (1867-1906) ordered himself a 32-cm photographically equipped Newtonian reflector from George Calver. He housed it in a observatory in Trincomalee and did significant research on Jupiter which he published in the Monthly Notices of the Royal Astronomical Society (Molesworth 1905). The telescope was moved after his death to Colombo. I had the opportunity to use this telescope when attending the University of Ceylon. A small 10-cm telescope gifted to Royal College which I attended had got me interested in astronomy. I was also encouraged by regular meetings of the Ceylon Astronomical Association founded in 1959 with Arthur Clarke as Patron. He has contributed very positively to encouraging an interest in astronomy in Sri Lanka. A 25-cm telescope was set up by Herschel Gunawardena at the Colombo Observatory. There was clearly sufficient interest in astronomy in Sri Lanka to initiate serious amateur observations and to motivate a few of us to take up astronomy as a career (http://lakdiva.com/astronomers/), even 25 years ago.

I also know of many astronomers with special interest in Sri Lanka. In 1975, Prof. Tom Gehrels of the University of Arizona, who had been enchanted by Ceylon in 1945, offered a gift of a 1.78-m telescope mirror (Gehrels 1984). Sufficient local interest in the project was very wisely required to build the rest. With no good site to ensure a useful return on the required investment, the telescope was never built. Sites which were in the dry zone were politically unsafe at that time and are even more so today, since it is that part of the country which is currently in a state of civil war. Sites of high altitude in the wet zone had over $75 \%$ of the days cloudy.

In the mid-1980s, the growth of instant electronic communication started opening the way for doing research over the Internet as we know it today. Interest of Lankans abroad in this new medium of communication was simulated by a need for news about Sri Lanka which was hardly covered in the foreign press. An informal e-mail group SLnet was formed in 1988 to exchange news, and Lankans were among the first dozen countries to form a Usernet group soc.culture.srilanka in 1989. A non-profit organization LAcNet was formed in 1991 which maintained a dial-up e-mail service till Lanka was connected directly to the Internet in 1995. One of the primary aims of this organization is to promote academic collaboration over the Internet between students in Sri Lanka and Lankan scientists overseas. In 1991/92, I spent a sabbatical year at the Institute for Fundamental Studies (IFS) in Kandy, Sri Lanka, exploring the possibility of doing astronomical research from Sri Lanka aided by the Internet which still needs to be funded properly.

Around the same time, IFS was offered a $45-\mathrm{cm}$ telescope by Japan. Being the only professional astronomer resident in the island at that time, I tried in vain to negotiate that most of the aid should be used to set up an Internet connection into Lanka and a computer laboratory which could be used for active astronomical research. A small mobile telescope (even up to 36-cm aperture can be an off-the-shelf item and relatively inexpensive) would have been more useful to serve the purpose of inspiring students to study astronomy. Although I stalled 
the telescope project, it restarted after I left Sri Lanka. A 45-cm telescope gifted by Japan was inaugurated at the UN/ESA Workshop on Basic Space Science, held in Colombo in Jan 1996. It was equipped with a photometer which is useless at the brightly lit urban site. More recently a CCD was gifted, the images from which will probably suffer from the instability of the telescope (mounted on the fourth floor of a building) caused by trucks on the nearby highway.

It remains as I predicted practically unused and, as far as I know, used only as an exhibit for visiting schoolchildren. For maintenance and supervision it costs about U.S. $\$ 5000$ per year of the very limited resources available for astronomy. For more details see Snowden (2001). Back in 1964 the Sri Lankan government, using a gift from East Germany, set up a Zeiss Planetarium as a part of an Industrial Exhibition. For a long time that was considered by many politicians as funding the needs of astronomical research. The new "white elephant" will similarly hurt more than help any future effort to invest in needs of astronomical research in Sri Lanka.

That the 45-cm telescope has served no purpose for astronomical research or education in Lanka is not entirely due to a lack of interest. Bureaucrats in the host institution, who have no motivation to use the instrument themselves, do not even allow access to it by students from the nearby university or to local amateurs since the telescope is listed as costing $\$ 300,000$ of aid to Sri Lanka. An active amateur group located nearby at the Institute for Integral Education run by Father Mervyn Fernando could be learning on this instrument. It is sadly ironic that, instead, they received a gift of a $20-\mathrm{cm}$ telescope from George Coyne, the Director of the Vatican Observatory. That this smaller telescope can be moved to a site reasonable for observation helps the group to use it more frequently.

Personal computers are now freely available in Sri Lanka at reasonable cost. Internet bandwidth is what is most lacking. Future plans are for a $0.5 \mathrm{Mbit}$ line which seems clearly inadequate for supporting a user community of very much more than 25 users. The academic bandwidth into the whole country is less than what would be considered acceptable even for a small university in the U.S.A.

\section{Conclusion}

A small telescope installed in a bright urban setting may not be the best way to develop a small astronomy research program in a developing nation. A decision to set up such an instrument needs to be based on the availability of a reasonable site, and the recommendations of local astronomers with expertise in both astronomy and the aspirations of the local user community.

In the global village we now live in, international collaborations are practical if the developing nation is properly networked. The Internet also gives you access to enormous data archives with the latest observations for quantitative analysis with computer systems which are now not very expensive. It is truly an optimum time for any developing nation interested in astronomy research to seriously consider doing it via the Internet.

Astronomical research in Sri Lanka is probably typical of many developing countries. Although there is a strong amateur astronomy interest in the country there are less than a dozen Lankans who are professional astronomers; all of them 
are doing research outside Sri Lanka. Could they or more recent graduates be attracted back? I think this is a clear possibility if the infrastructure needed for research is set up in Sri Lanka. Linking Sri Lanka to the Internet with an electronic superhighway is not the only need, but it is clearly the minimum before one can consider the possibility of active research seriously.

Acknowledgments. I wish thank the organizing committee for inviting me to give this contribution and for the financial support in attending the 24th IAU general assembly in Manchester, U.K.

\section{References}

Gehrels, T. 1984 in Fundamental Studies and the Future of Science ed. C. Wickramasinghe (Cardiff:University College) p.377.

Gehrels, T. 1988 On the Glassy Sea: An Astronomer's Jouney, AIP, New York, p. 236.

Molesworth, P. B. 1905 MNRAS65 691

Ratnatunga, K. U., Ostrander, E. J., Griffiths, R. E., \& Im, M. 1995 ApJ453 L5

Ratnatunga, K. U., Griffiths, R. E. \& Ostrander, E. J. 1999, AJ118 86

Snowden, M. S., 2001, this volume, pp. 266-275.

MacKenzie, D. 2000, New Scientist 22-April 2000.

\section{Appendix}

I give below a sampling of the major Internet sites with URL's of astronomical databases. A more complete and current list could be obtained from AstroWeb (http://cdsweb.u-strasbg.fr/astroweb.html).

Centre de Donnes astronomiques de Strasbourg (CDS http://cdsweb.u-strasbg.fr/) which hosts Set of Identifications, Measurements, and Bibliography for Astronomical Data (SIMBAD http://simbad.u-strasbg.fr/Simbad/) an astronomical database provides basic data, cross-identifications and bibliography for currently about 3 million astronomical objects outside the solar system.

VizieR (CDS http://vizier.u-strasbg.fr/)provides on line, a library of computer-readable astronomical catalogues and data tables with documentation. It is mirrored in Astronomy Data Centers in USA (ADC http://adc.gsfc.nasa.gov/), Japan (ADAC http://adac.mtk.nao.ac.jp/), and India (IUCAA http://www.iucaa.ernet.in/)

High Energy Astrophysics Science Archive Research Center (HEASARC http://heasarc.gsfc.nasa.gov/). A source of $\gamma$-ray, X-ray, and extreme ultraviolet observations of cosmic sources with direct links associated support facilities. It hosts a Virtual Observatory (SkyView http://skyview.gsfc.nasa.gov/) for generating images of any part of the sky at wavelengths in all regimes from Radio to Gamma-Rays. 
The Hubble Data Archive (HDA http://archive.stsci.edu/) which also supports a Multi-mission Archive (MAST http://archive.stsci.edu/mast.html) with a variety of astronomical data archives, with focus in the optical, ultraviolet, and near-infrared parts of the spectrum.

Infrared Processing and Analysis Center (IPAC http://www.ipac.caltech.edu/) maintain infrared data archives and access tools and hosts the NASA Extra-galactic Database (NED http://nedwww.ipac.caltech.edu/) with over 3 million objects and 150 thousand red-shifts.

Planetary Data System (PDS http://pds.jpl.nasa.gov/) archives digital data from past and present NASA planetary missions, astronomical observations, and laboratory measurements. All data is classified Technology and Software Publicly Available (TSPA) to be exported outside the United States.

Canadian Astronomy Data Center (CADC http://cadcwww.dao.nrc.ca/) which host the archive observations since 1990 from the Canada-France-Hawaii 4-meter Telescope on Mauna Kea, Hawaii, (CFHT

http://cadcwww.dao.nrc.ca/cfht/). Links are provided to similar archives setup at many major ground-based observatories worldwide using CADC software and expertise.

Sloan Digital Sky Survey (SDSS http://www.sdss.org/) which will systematically map one fourth of the sky to probe the large-scale structure of the universe. The survey will produce a catalog of roughly 100 million objects, with red-shifts to more than a million galaxies and quasars.

Astrophysics Data System (ADS http://adswww.harvard.edu/) A NASA-funded Abstract Service which provides access to over two million astronomy related abstracts which can be searched by author, title, object name, or keywords. It has also made agreements with publishes of astronomical journals to provide links to scanned images of over 40,000 articles appearing in most of the major astronomical journals and over 3 years old.

Physics e-Print archive (XXX http://xxx.lanl.gov/) which includes astrophysics (astro-ph). Mirror sites are being established all over the world. Replacing postal distribution of preprints.

AAS job Register (AAS http://www.aas.org/JobRegister/aasjobs.html) A monthly posting of worldwide astronomy related jobs maintained by the American Astronomical Society.

Digitized Sky Survey (DSS http://stdatu.stsci.edu/dss/) This comprises a set of all-sky photographic surveys conducted with the Palomar and UK 48-inch Schmidt telescopes and scanned using a PDS microdensitometer to scale of about 1.0 to 1.7 arcseconds per pixel.

Global Network of Astronomical Telescopes (GNAT http://www.gnat.org/) A non-profit organization dedicated as an information source for all those interested in research and education using relatively small astronomical telescopes.

International Dark-Sky Association (IDA http://www.darksky.org/) A non-profit organization with a goal to stopping the adverse environmental impact of light pollution and space debris through education about the value and effectiveness of quality nighttime lighting. 
International Astronomical Union (IAU http://www.iau.org/) Announcements, Information bulletin and services.

Discussion Martinez agreed that a good Internet connection may be preferable to the building of a "research-grade" telescope but thought that the promotion of linkages between scientists in developed and developing countries is also essential. Without such links, scientists in developing countries will not be in a position to conduct competitive research and the gap in research capacity will get wider. Providing access to our data is not sufficient; we have to provide our colleagues with our time, expertise and research programmes as well. Ratnatunga agreed but thought that short visits would create the links which could be maintained over the Internet by e-mail.

Al-Sabti said that reserarch based on the Internet was useful but should not be a substitute for building telescopes and observatories. Doing astronomy through the Internet will create dependency on advanced countries similar to that of the developing world on the technology of the West. Ratnatunga replied that technology of instrumentation was only one aspect of the problem. Analysis software has to be developed and this can be done with only a PC. Percy agreed about the Internet but pointed out that good research can be carried out with a telescope costing $\$ 10,000$ on an urban site (as Snowden, p. 269, had pointed out). Ratnatunga replied that if he had $\$ 300,000$ to spend, he would spend $\$$ 10,000 on the telescope and the rest to establish good Internet connections!

Kochhar said that, by extending Ratnatunga's argument, one need not cook food at home because cooked food can be bought from the market. Dworetsky suggested that the analogy should be that a fine picnic has been set out; the food is free, but bring your own utensils. In every hundredth cake there is a gold nugget hidden. Ratnatunga thought this much the better analogy. Schreuder suggested that a summary might be: telescopes are better for education, the Internet for research. Ratnatunga said that the Internet can be used for education as well. Developing software for analysis is educational and data from the Internet are no different from those from a telescope. 